EPJ manuscript No.

(will be inserted by the editor)

\title{
Meson-Baryon s-wave Resonances with Strangeness -3
}

\author{
C. García-Recio, J. Nieves, and L. L. Salcedo \\ Departamento de Física Atómica, Molecular y Nuclear, Universidad de Granada, E-18071 Granada, Spain \\ Received: date / Revised version: date
}

\begin{abstract}
Starting from a consistent SU(6) extension of the Weinberg-Tomozawa (WT) meson-baryon chiral Lagrangian (Phys. Rev. D74 (2006) 034025), we study the s-wave meson-baryon resonances in the strangeness $S=-3$ and negative parity sectors. Those resonances are generated by solving the BetheSalpeter equation with the WT interaction used as kernel. The considered mesons are those of the 35$\mathrm{SU}(6)$-plet, which includes the pseudoscalar (PS) octet of pions and the vector (V) nonet of the rho meson. For baryons we consider the 56-SU(6)-plet, made of the $1 / 2^{+}$octet of the nucleon and the $3 / 2^{+}$decuplet of the Delta. Quantum numbers $I\left(J^{P}\right)=0\left(3 / 2^{-}\right)$are suggested for the experimental resonances $\Omega^{*}(2250)^{-}$ and $\Omega^{*}(2380)^{-}$. Among other, resonances with $I=1$ are found, which minimal quark content is $s s s \bar{l} l^{\prime}$, being $s$ the strange quark and $l, l^{\prime}$ any of the the light up or down quarks. A clear signal for such a pentaquark would be a baryonic resonance with strangeness -3 and electric charge of -2 or 0 , in proton charge units. We suggest looking for $K^{-} \Xi^{-}$resonances with masses around 2100 and $2240 \mathrm{MeV}$ in the sector $1\left(1 / 2^{-}\right)$, and for $\pi^{ \pm} \Omega^{-}$and $K^{-} \Xi^{*-}$ resonances with masses around $2260 \mathrm{MeV}$ in the sector $1\left(3 / 2^{-}\right)$.
\end{abstract}

PACS. 11.30.Hv Flavor symmetries - 11.30.Ly Other internal and higher symmetries - 11.10.St Bound and unstable states; Bethe-Salpeter equations - 11.30.Rd Chiral symmetries - 11.80.Gw Multichannel scattering

\section{Introduction}

Using a spin-flavor-SU(6) extended Weinberg-Tomozawa (WT) meson-baryon interaction ${ }^{1}$ [1, we study the s-wave resonances with strangeness $S=-3$, isospin $\mathrm{I}=0,1$ and spin-parity $\mathrm{J}^{P}=1 / 2^{-}, 3 / 2^{-}, 5 / 2^{-}$. The resonances are generated by solving the Bethe-Salpeter equation with the extended WT meson-baryon interaction used as a kernel. In this model, the involved mesons are those of the 35$\mathrm{SU}(6)$-plet $=8_{1} \oplus 8_{3} \oplus 1_{3}$, which includes the PS meson octet of the pions, $(\pi, \eta, K, \bar{K}) \in 8_{1}$ and the $\mathrm{V}$ nonet of the rho meson, $\left(\rho, \omega, \phi, K^{*}, \bar{K}^{*}\right) \in 8_{3} \oplus 1_{3}$. We approximate the $\eta$ meson as the isospin singlet state of the PS SU(3)-octet. For the $\omega$ and $\phi$ vector mesons, we assume ideal mixing among the $\mathrm{V}$ singlet and octet mesons. The baryons are those of the 56-SU(6)-plet $=8_{2} \oplus 10_{4}$, which contains the $1 / 2^{+}$octet of the $\mathrm{N}(N, \Lambda, \Sigma, \Xi)$ and the $3 / 2^{+}$decuplet of the $\Delta\left(\Delta, \Sigma^{*}, \Xi^{*}, \Omega\right)$. Masses, widths and couplings of the resonances found are calculated and, when possible, comparison with experimental ones is attempted.

Unitary extensions of chiral perturbation theory to study meson-baryon interactions using a coupled channel scheme were introduced some time ago 3 . They have been successfully applied in the theoretical microscopical description of meson-baryon scattering and of well

\footnotetext{
Send offprint requests to:

1 This WT interaction has also been extended to arbitrary number of colors and flavors in ref. 2]
}

known lowest lying baryon resonances, which were shown to be dynamically generated. Thus different $J^{P}=1 / 2^{-}$ $s$-wave resonances $\left(\subset 8_{\pi} \times 8_{N}\right.$, made of PS mesons of the pion octet and of the baryons of the nucleon octet) like $N^{*}(1535), \Lambda(1405), \Lambda(1670), \Sigma(1620)$ and $\Xi(1620)$ 4 and, more recently, the $J^{P}=3 / 2^{-} d$-wave resonances ( $\subset 8_{\pi} \times 10_{\Delta}$, made of PS mesons of the pion octet and of the baryons of the delta decuplet) like $\Lambda(1520), \Sigma(1670)$ and $\Xi(1820)$ [5] have been found and their properties studied. Those previous chiral Bethe-Salpeter coupled channels unitary approaches using the WT kernel have included hadron multiplets belonging to the flavor SU(3) irreducible representations. In the case of mesons, the only ingredient has been the octet of PS mesons.

\section{Model}

Motivations for extending the previous SU(3) based models to a $\mathrm{SU}(6)$ extended model are the following. First, in the large $N_{c}$ limit, $8_{N}$ and $10_{\Delta}$ are degenerated and form a 56-multiplet of spin-flavor SU(6). Second, vector mesons do exist, interact and couple to baryons. Third, there are baryonic resonances decaying to a PS meson and a baryon, but also to a $\mathrm{V}$ meson and a baryon, for instance, the strangeness -3 resonance $\Omega^{*}(2380)^{-}$decays to $K^{-} \Xi^{* 0}$ and to $\bar{K}^{* 0} \Xi^{-}$with similar strengths and of the same order as the other known decay mode $\left(\bar{K}^{-} \pi^{0} \Xi^{-}\right)$[8]. All of these call for a 'SU(6)' model which deals all together with 
C. García-Recio et al.: Meson-Baryon s-wave Resonances with Strangeness -3

the 56-baryons and 35-mesons like that of ref. 11. We consider this spin-flavor symmetric scenario as a reasonable first approach. In the 'SU(6)' approach the interacting kernel $V^{I Y J}$ for a sector with hypercharge $Y$ (strangeness plus one for baryons), isospin $I$ and spin $J$ is given by:

$$
\begin{gathered}
\left\langle m_{i}, B_{i}\left|V^{I Y J}(s)\right| m_{j}, B_{j}\right\rangle=D_{i j}^{I Y J} \frac{2 \sqrt{s}-M_{B_{i}}-M_{B_{j}}}{4 f^{2}} \\
m_{i}, m_{j} \in \mathbf{3 5}, B_{i}, B_{j} \in \mathbf{5 6}, \quad D^{I Y J}=\sum_{\nu} \bar{\lambda}_{\nu} \hat{P}_{\nu}^{I Y J} \\
\bar{\lambda}_{\mathbf{5 6}}=-12, \bar{\lambda}_{\mathbf{7 0}}=-18, \quad \bar{\lambda}_{\mathbf{7 0 0}}=6, \bar{\lambda}_{\mathbf{1 1 3 4}}=-2,
\end{gathered}
$$

$\hat{P}_{\nu}^{I Y J}$ is the projector of the meson-baryon into the $\nu$ $\mathrm{SU}(6)$-representation, and $\nu$ runs over 56, 70, 700, 1134 because $\mathbf{3 5} \otimes \mathbf{5 6}=\mathbf{5 6} \oplus \mathbf{7 0} \oplus \mathbf{7 0 0} \oplus \mathbf{1 1 3 4}$.

The $\bar{\lambda}_{\nu}$ positive (negative) means that in channel $\nu$ the meson-baryon interaction is repulsive (attractive). When this kernel is restricted to $m_{i}, m_{j}$ being only PS mesons, it coincides with the 'SU(3)' lowest order WT kernels previously used in refs. 4.5.

Note that $D$ is $\mathrm{SU}(6)$ invariant, this symmetry being explicitly broken by the different masses of baryons and mesons. In addition we replace the $f^{2}$ of the interaction by $f_{m_{i}} f_{m_{j}}$ for the $i j$ matrix element. We use $f_{\pi}=92.4 \mathrm{MeV}$, $f_{K}=1.15 f_{\pi}, f_{\eta}=1.2 f_{\pi}$ [7], and $f_{K^{*}}=f_{K}, f_{\rho}=f_{\pi}$, $f_{\omega}=f_{\phi}=f_{\eta}$. This interaction is used to solve the BetheSalpeter coupled channel equation for the meson-baryon $T$-matrix

$$
T^{-1}(s)=V^{-1}(s)-J(s)
$$

where $J(s)$ is the diagonal matrix of the meson-baryon loop functions 4,5]. For each channel $\left(m_{i}, B_{i}\right)$ it is ultraviolet regularized by subtracting a constant so that $J\left(s=m_{m_{i}}{ }^{2}+M_{B_{i}}{ }^{2}\right)=0$.

We test that the inclusion of the new 'SU(6)' channels (those involving $\mathrm{V}$ mesons not included in the ' $\mathrm{SU}(3)$ ' calculations) does not spoil previous results which were successful. See ref. [6] for comparison in the sector $(S=$ $\left.-1, I=0, J^{P}=1 / 2^{-}\right)$.

\section{Results}

We solve the coupled-channel Bethe-Salpeter equation and look for the $T$-matrix poles in the second Riemann sheet. Close to a pole the $T$-matrix behaves as

$$
T_{i j} \sim \frac{g_{i} g_{j}}{\sqrt{s}-\left(M_{R}-i \Gamma_{R} / 2\right)}
$$

and the position of the pole and its residue define the mass $M_{R}$, width $\Gamma_{R}$ and complex coupling constants $g_{i}$ to different $i$ channels of the found resonance.

All the calculations are done neglecting the widths of the baryons of the decuplet and of the $\mathrm{V}$ mesons. When the orbital angular momentum of the meson-baryon system is zero, the odd parity $S=-3$ resonances formed by coupling 35-mesons to 56-baryons can have the following $I\left(J^{P}\right)$ quantum numbers: $0\left(1 / 2^{-}\right), 0\left(3 / 2^{-}\right), 0\left(5 / 2^{-}\right)$, $1\left(1 / 2^{-}\right), 1\left(3 / 2^{-}\right)$and $1\left(5 / 2^{-}\right)$. The mass, width and absolute values of the coupling constants of the resonances found for each of those sectors are shown in tables [1] 2 [3] 4] [5] and 6] respectively. Resonances with width below $125 \mathrm{MeV}$ are displayed in fig. 1.

Several $(S=-3, I=1)$ resonances have been found (tables 4 [ 5] and 6] and full symbols in fig. 1). These resonances have, at least, three $s$ quarks to provide strangeness $S=-3$ and a pair of light $(u$ or $d$ ) quark-antiquark to achive isospin $I=1$ quantum number. Hence, those dynamically generated $(S=-3, I=1)$ resonances are pentaquarks and in principle they could be empirically spotted very easily. A clear signal of them would be a resonance with strangeness $S=-3$ and electric charge $Q=-2$ (this is $I_{z}=-1$ ) with minimal quark content $s s s d \bar{u}$. Another clear signature would be a $S=-3$ and $Q=0$ resonance with a minimal quark content of sssu $\bar{d}$. From the tables, the best ways for observing pentaquarks would be, in the sector $I\left(J^{P}\right)=1\left(1 / 2^{-}\right)$looking for $K^{-} \Xi^{-}$resonances with masses around 2100 and $2240 \mathrm{MeV}$, and in the sector $I\left(J^{P}\right)=1\left(3 / 2^{-}\right)$looking for $\pi^{ \pm} \Omega^{-}$and $K^{-} \Xi^{*-}$ resonances with masses around $2260 \mathrm{MeV}$.

All the experimentally known $\Omega^{*}$ resonances are listed in table[7 Tentatively, we identify the experimental isoscalar $\Omega^{*}(2250)^{-}$to the theoretical $\Omega^{*}(2265)^{-}$of sector $0\left(3 / 2^{-}\right)$, because of the relative closeness of masses and widths and, also, of observed decay channels. Likewise, the experimental $\Omega^{*}(2380)^{-}$could be assigned to the found $\Omega^{*}(2343)^{-}$ of sector $0\left(3 / 2^{-}\right)$. We do not find a clear assignment for the experimental $\Omega^{*}(2470)$ because it decays to $\Omega^{-} \pi^{+} \pi^{-}$, and the resonances that we find around that energy have not the possibility of decaying to such channel.

More details will be given elsewhere.

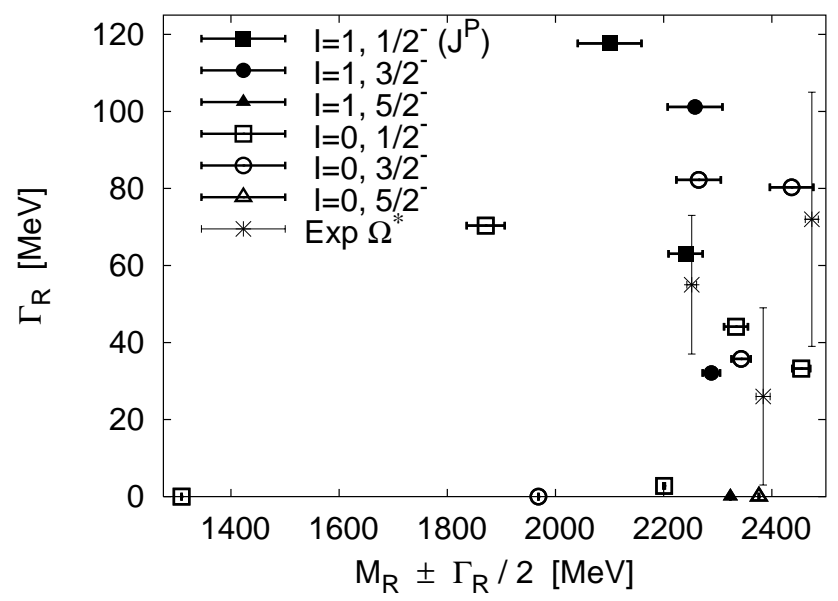

Fig. 1. Spin-parity $J^{P}=\frac{1}{2}^{-}, \frac{3}{2}^{-}$and $\frac{5}{2}^{-}$resonance properties in the $I=0$ (empty symbols) and $I=1$ (filled symbols), $S=$ $-3(Y=-2)$ sectors. The points with error-bars are defined from the masses $\left(M_{R}\right)$ and widths $\left(\Gamma_{R}\right)$ of the found resonances as $\left(M_{R} \pm \Gamma_{R} / 2, \Gamma_{R}\right)$. The experimental $\Omega^{*}$ resonance masses and widths with their error bars are from refs. 78910 
Table 1. Masses, widths and absolute values of coupling constants for each channel, in the sector $I=0, J^{P}=1 / 2^{-}$and $S=-3$. The underlining indicates open channels. 'SU(6)' stands for the full $\mathbf{3 5} \times \mathbf{5 6}$ result. 'SU(3)' for the $\mathbf{8} \times \mathbf{8}$ result.

\begin{tabular}{|cc||ccccc|}
\hline \multicolumn{7}{|c|}{$I=0, J^{P}=1 / 2^{-}, ' \mathrm{SU}(6)$} \\
\hline $\begin{array}{c}\mathrm{M}_{R} \\
{[\mathrm{MeV}]}\end{array}$ & $\Gamma_{R}$ & $\bar{K} \Xi$ & $\bar{K}^{*} \Xi$ & $\bar{K}^{*} \Xi^{*}$ & $\omega \Omega$ & $\phi \Omega$ \\
\hline 1309 & 00 & 1.22 & 0.61 & 3.27 & 0.17 & 5.78 \\
1871 & 70 & $\underline{1.57}$ & 3.63 & 4.17 & 1.39 & 3.23 \\
2201 & 2.8 & $\underline{0.22}$ & 1.63 & 2.36 & 0.48 & 1.38 \\
2334 & 44 & $\underline{0.76}$ & $\underline{0.37}$ & 0.66 & 3.44 & 1.08 \\
2454 & 33 & $\underline{0.38}$ & $\underline{0.22}$ & $\underline{0.97}$ & 0.18 & 4.32 \\
\hline- & - & - & & & & 'SU(3)' \\
\hline
\end{tabular}

Table 2. Same as Table 1 for the sector $I=0, J^{P}=3 / 2^{-}$.

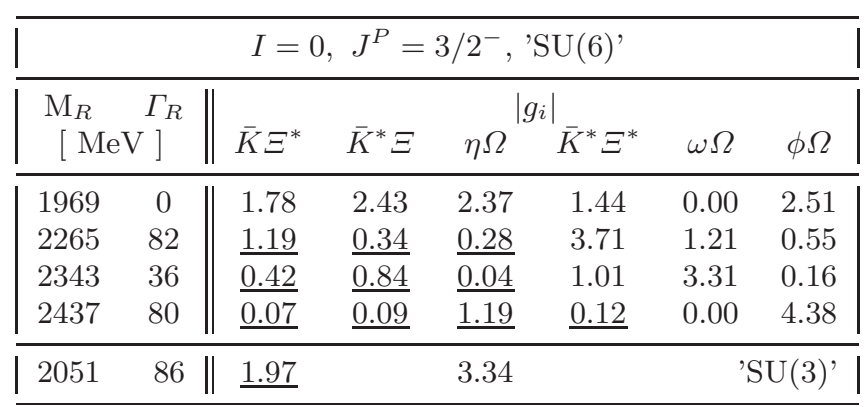

Table 3. Same as Table $\prod$ for the sector $I=0, J^{P}=5 / 2^{-}$.

\begin{tabular}{|c|c|c|c|}
\hline \multicolumn{4}{|c|}{$I=0, J^{P}=5 / 2^{-},{ }^{\prime} \mathrm{SU}(6) '$} \\
\hline $\begin{array}{c}\mathrm{M}_{R} \quad \Gamma_{R} \\
{[\mathrm{MeV}]}\end{array}$ & $\bar{K}^{*} \Xi^{*}$ & $\begin{array}{l}\left|g_{i}\right| \\
\omega \Omega\end{array}$ & $\phi \Omega$ \\
\hline 2376 & 1.41 & 2.78 & 0.00 \\
\hline
\end{tabular}

Table 4. Same as Table $\prod$ for the sector $I=1, J^{P}=1 / 2^{-}$.

\begin{tabular}{|c|c|c|c|c|c|}
\hline \multicolumn{6}{|c|}{$I=1, J^{P}=1 / 2^{-},{ }^{\prime} \mathrm{SU}(6)^{\prime}$} \\
\hline $\begin{array}{l}\mathrm{M}_{R} \\
\quad[\mathrm{Me}\end{array}$ & & $\bar{K} \Xi$ & $\bar{K}^{*} \Xi$ & $\bar{K}^{*} \Xi^{*}$ & $\rho \Omega$ \\
\hline 2100 & 118 & 1.47 & 3.39 & 1.1 & 2.1 \\
\hline 2241 & 63 & $\overline{0.88}$ & $\underline{0.87}$ & 1.67 & 3.87 \\
\hline- & - & - & & & $\mathrm{U}(3)^{\prime}$ \\
\hline
\end{tabular}

We acknowledge discussions with Drs. Angels Ramos and Manuel J. Vicente-Vacas. This work was supported by DGI, FEDER, UE and Junta de Andalucía funds (FIS2005-00810, HPRN-CT2002-00311, FQM225).

\section{References}

1. C. Garcia-Recio, J. Nieves and L. L. Salcedo, Phys. Rev. D 74 (2006) 034025.
Table 5. Same as Table 1 for the sector $I=1, J^{P}=3 / 2^{-}$.

\begin{tabular}{|c|c|c|c|c|c|c|}
\hline \multicolumn{7}{|c|}{$I=1, J^{P}=3 / 2^{-},{ }^{\prime} \mathrm{SU}(6)^{\prime}$} \\
\hline $\begin{array}{l}\mathrm{M}_{R} \\
\quad[\mathrm{MeV}\end{array}$ & & $\pi \Omega$ & $\bar{K} \Xi^{*}$ & $\frac{\left|g_{i}\right|}{\bar{K}^{*} \Xi}$ & $\bar{K}^{*} \Xi^{*}$ & $\rho \Omega$ \\
\hline 2018 & 267 & $\underline{2.19}$ & 1.64 & 2.14 & 1.75 & 0.25 \\
\hline 2258 & 101 & $\underline{0.54}$ & $\underline{1.23}$ & $\underline{0.13}$ & 3.19 & 2.33 \\
\hline 2288 & 32 & $\underline{0.33}$ & $\underline{0.17}$ & $\underline{0.93}$ & 2.29 & 3.11 \\
\hline 2146 & 359 & $\underline{2.30}$ & 2.47 & & & $\mathrm{U}(3)^{\prime}$ \\
\hline
\end{tabular}

Table 6. Same as Table 1 for the sector $I=1, J^{P}=5 / 2^{-}$.

\begin{tabular}{|c|c|c|}
\hline \multicolumn{3}{|c|}{$I=1, J^{P}=5 / 2^{-},{ }^{\prime} \mathrm{SU}(6)^{\prime}$} \\
\hline $\begin{array}{c}\mathrm{M}_{R} \quad \Gamma_{R} \\
{[\mathrm{MeV}]}\end{array}$ & $\bar{K}^{*} \Xi^{\mid} \mid g_{i}$ & $\rho \Omega$ \\
\hline 2324 & 1.79 & 3.09 \\
\hline
\end{tabular}

Table 7. Experimentally known $\Omega^{*}$ resonances 78 , 10]. The branching ratios are relative.

\begin{tabular}{c|cc|c|l}
\hline $\begin{array}{c}\text { Resonance } \\
I(J)^{P}\end{array}$ & $\begin{array}{c}\text { Mass } \\
{[\mathrm{MeV}]}\end{array}$ & $\begin{array}{c}\text { Width } \\
\text { modes }\end{array}$ & $\begin{array}{l}\text { Branching } \\
\text { ratios }\end{array}$ \\
\hline $\begin{array}{c}\Omega^{*}(2250)^{-} \\
0\left(?^{?}\right)^{* * *}\end{array}$ & $2252 \pm 9$ & $55 \pm 18$ & $\Xi^{-} \pi^{+} K^{-}$ & 1 \\
$\Omega^{* 0} K^{-}$ & $0.7 \pm 0.2$ \\
\hline$\Omega^{*}(2380)^{-}$ & $2384 \pm 13$ & $26 \pm 23$ & $\Xi^{-} \pi^{+} K^{-}$ & 1 \\
& & & $\Xi^{* 0} K^{-}$ & $<0.4$ \\
& & & $\Xi^{-} \bar{K}^{*}$ & $0.5 \pm 0.3$ \\
\hline$\Omega^{*}(2470)^{-}$ & $2474 \pm 12$ & $72 \pm 33$ & $\Omega^{-} \pi^{+} \pi^{-}$ & \\
$?\left(?^{?}\right)^{* *}$ & & & & \\
\hline
\end{tabular}

2. C. Garcia-Recio, J. Nieves and L. L. Salcedo, Phys. Rev. D 74 (2006) 036004; Ibidem, hep-ph/0610204

3. N. Kaiser, P. B. Siegel and W. Weise, Nucl. Phys. A 594 (1995) 325; N. Kaiser, T. Waas and W. Weise, Nucl. Phys. A 612 (1997) 297; E. Oset and A. Ramos, Nucl. Phys. A 635 (1998) 99; J. A. Oller and U. G. Meissner, Phys. Lett. B 500 (2001) 263.

4. J. Nieves and E. Ruiz Arriola, Phys. Rev. D 64 (2001) 116008; T. Inoue, E. Oset and M. J. Vicente Vacas, Phys. Rev. C 65 (2002) 035204; D. Jido et al, Nucl. Phys. A 725 (2003) 181; C. Garcia-Recio et al, Phys. Rev. D 67 (2003) 076009; C. Garcia-Recio, M. F. M. Lutz and J. Nieves, Phys. Lett. B 582 (2004) 49; J. A. Oller, arXiv:hep-ph/0603134

5. E. E. Kolomeitsev and M. F. M. Lutz, Phys. Lett. B 585 (2004) 243; S. Sarkar, E. Oset and M. J. Vicente Vacas, Nucl. Phys. A 750 (2005) 294; S. Sarkar, E. Oset and M. J. Vicente Vacas, Eur. Phys. J. A 24 (2005) 287; L. Roca, S. Sarkar, V. K. Magas and E. Oset, Phys. Rev. C 73 (2006) 045208. 6. C. Garcia-Recio, J. Nieves and L. L. Salcedo, arXiv:hep-ph/0610127

7. W.-M. Yao et al. (Particle Data Group), J. Phys. G 33 (2006) 1.

8. S. F. Biagi et al., Z. Phys. C 31 (1986) 33.

9. D. Aston et al., Phys. Lett. B 194 (1987) 579.

10. D. Aston et al., Phys. Lett. B 215 (1988) 799. 